\title{
Influencia de la Goma de Tara (Caesalpinia spinosa) como Ayudante en el Proceso de Coagulación-Floculación para la Remoción de Turbidez de una Suspensión Artificial de Bentonita
}

\author{
Jappsem J. Valeriano-Mamani y Rodrigo A. Matos-Chamorro \\ Facultad de Ingeniería y Arquitectura, Universidad Peruana Unión, Lima - Perú. (e-mail: japsem1@gmail.com; \\ amatosch@upeu.edu.pe)
}

Recibido Dic. 3, 2018; Aceptado Feb. 6, 2019; Versión final Abr. 15, 2019, Publicado Oct. 2019

\begin{abstract}
Resumen
El objetivo de la investigación fue determinar la influencia de la goma de tara (Caesalpinia spinosa) como ayudante de coagulación en el proceso de coagulación-floculación para la remoción de turbidez de una suspensión artificial de bentonita. El coagulante primario usado fue el sulfato de aluminio tipo A y el ayudante de coagulación fue goma de tara. Se utilizó la metodología de prueba de jarras programando velocidades de 300 revoluciones por minuto (RPM) y 5 segundos de acción para la mezcla rápida, 40 RPM y 20 minutos para la floculación, y 0 RPM por espacio de 10 minutos para la sedimentación. Se representan aguas de alta y baja turbidez de 400 unidades nefelométricas de turbidez (UNT) y 30 UNT respectivamente. Los resultados obtenidos con sulfato de aluminio tipo A sin ayudante de coagulación arroja resultados de turbidez residual de 1.09 UNT para muestras de turbidez alta y 0.57 UNT para la muestra de turbidez baja. Añadiendo goma de tara se redujo a 0.40 y 0.32 UNT de turbidez residual para los dos casos mencionados. También se logró la reducción de $40 \%$ de sulfato de aluminio para las pruebas con la muestra de 30 UNT. La goma de tara, como ayudante de coagulación en el proceso de coagulación-floculación mejora la reducción de la turbidez residual en $63.3 \%$ en el caso de 400 UNT y de $56 \%$ en el caso de 30 UNT.
\end{abstract}

\section{Influence of Tara (Caesalpinia spinosa) Gum as an Aid in the Coagulation-Flocculation Process to Remove the Turbidity of an Artificial Suspension of Bentonite}

\begin{abstract}
The objective of this study was to determine the influence of tara gum (Caesalpinia spinosa) as a coagulation aid in the coagulation-flocculation process to remove the turbidity of an artificial suspension of bentonite. The primary coagulant used was aluminum sulfate type $A$ and the coagulation assistant was tara gum. The jar test methodology was used, at speeds of 300 revolutions per minute (RPM) for 5 seconds in the fast mixing stage, 40 RPM for 20 minutes in the flocculation stage, and 0 RPM for 10 minutes in the sedimentation stage. High and low turbidity waters of 400 nephelometric turbidity units (NTU) and 30 NTU were analyzed. The results obtained with type A aluminum sulfate without a coagulation aid give residual turbidity results of 1.09 NTU for high turbidity samples and 0.57 NTU for the low turbidity sample. Adding tara gum as a coagulation aid reduced the turbidity levels in the two sample groups to 0.40 and 0.32 NTU of residual turbidity respectively, as well as a $40 \%$ reduction of aluminum sulfate for the tests with the sample of 30 NTU. Tara gum, as a coagulation aid in the coagulation-flocculation process, improves the reduction of residual turbidity by $63.3 \%$ in the case of the 400 NTU sample group and $56 \%$ in the case of the 30 NTU sample group.
\end{abstract}

Keywords: tara gum; Caesalpinia spinosa; aluminum sulfate; turbidity; coagulation; flocculation 


\section{INTRODUCCIÓN}

Se ha observado un incremento de materia orgánica natural durante los últimos 10 a 20 años, éstas se encuentran en todas las aguas superficiales, subterráneas y del suelo en el suministro de agua cruda en varias áreas, incrementando la diversidad de micro contaminantes orgánicos, lo que tiene un efecto significativo en el tratamiento del agua potable (Matilainen et al., 2010; Yu et al., 2018). La remoción de los contaminantes en aguas naturales, es una de las tareas importantes que se realiza en el proceso de tratamiento de agua para consumo humano, las partículas coloidales que generan turbidez son elementos de dispersión de patógenos que afectan la salud a los pobladores (Joshi y Sahju, 2014). Díaz et al., (2007) y Yongabi (2010) mencionan que las aguas turbias está directamente relacionada a la incidencia de episodios gastrointestinales, cólera, fiebre tifoidea, disenterías, poliomielitis, hepatitis y salmonelosis, entre otras enfermedades, causadas por la presencia de virus, parásitos y bacterias presentes, como el Cryptosporidium (Abramovich et al., 2004), Vibrio cholerae, Escherichia coli, Salmonella (Díaz et al., 2007), etc. La Organización mundial de la salud (OMS, 2012) reporta que los niveles máximos de turbidez en el agua para consumo humano no deben exceder a una unidad nefelométrica de turbidez (1 UNT) para garantizar la ausencia de estos microorganismos. En el proceso de potabilización del agua, la clarificación es la eliminación de los materiales sólidos y coloidales (Restrepo, 2009), que involucra las fases de coagulación, donde se agregan compuestos químicos y polímeros sintéticos para aglutinar los sólidos en suspensión; la floculación, es la acumulación, por gravedad y filtrado de los flóculos, y la sedimentación, en la cual se retiran las partículas portadoras de bacterias que generan el color y la turbiedad del agua (Letterman et al., 1999; Aguirre et al., 2018). El compuesto químico más utilizado es el sulfato de aluminio (Barrenechea, 2004).

Investigaciones recientes han vinculado el uso de sulfato de aluminio para el tratamiento de agua con algunas enfermedades neurodegenerativas y neurotóxicas en seres vivos (Adedayo et al., 2019). Sin embargo, Gauthier et al., (2000) afirmaron que el aluminio presente en agua para consumo no está asociado de manera significativa con la enfermedad del Alzheimer en personas mayores de 70 años. Por otro lado, un estudio previo realizado por Wicklund et al., (1994) determinaron el incremento de aluminio en tejidos óseos, hepáticos y en el cerebro de ratas expuestas al agua para consumo humano. De la misma manera, Becaria et al., (2006) experimentando con animales de laboratorio, sugieren que el aluminio puede iniciar efectos inflamatorios u oxidativos a nivel neuronal aumentando el riesgo de incrementar los niveles de la Proteína Precursora del Amiloide (APP), la cual está presente en tejidos cerebrales de personas que sufren de la enfermedad del Alzheimer.

Por los problemas relacionados a la salud pública y al medio ambiente, ocasionados por el uso del sulfato de aluminio, investigaciones recientes han explorado otras sales basadas en metales (no sales de $\mathrm{Al}$ o $\mathrm{Fe}$ ) preparando coagulantes para el tratamiento de agua potable como el tetracloruro de circonio y titanio siendo efectivo para la eliminación de sustancias húmicas (HA y FA) cerca de sus puntos isoeléctricos (Jia, 2015), también se buscaron alternativas con compuestos orgánicos derivados de fuentes vegetales y animales, con funciones similares a los compuestos químicos. Fueron investigados diferentes compuestos orgánicos, entre ellos el Chitosan, extraído del exoesqueleto de moluscos (Katrivesis et al., 2019), las semillas de Moringa oleífera ( Preston et al., 2010), el extracto de Scirpus californicus (Yongabi, 2010), semillas de Plantago ovata (Shahriari et al., 2011), el Cochifloc, extraído de la penca de la tuna (Almendárez, 2004), extracto del Hylocereus lemairei (Mendoza et al., 2007), almidón de mandioca (Di Bernardo et al., 2002), almidón de plátano (Trujillo et al., 2014), polielectrolitos catiónicos, aniónicos y no iónicos (Bolto y Gregory, 2007). También el Centro Panamericano de Ingeniería Sanitaria y Ciencias del Ambiente, experimentó con polímeros naturales, como la goma arábiga, goma carragen, goma tragacanto, tunafloc, logrando eficiencias de remoción de turbidez inclusive mayores al 90\% (Kirchmer et al., 1975). Aguirre et al., (2018) reportan que las semillas Moringa oleifera logra una reducción de 95 y $92 \%$ de turbidez y de color a dosis de 1.5, 2.0 y $2.5 \mathrm{~g} /$ L. El coagulante obtenido de O. ficus-indica, logró reducir el $51.3,52.9$ y $57.2 \%$ de color, respectivamente, y alrededor del $62 \%$ de la turbidez a dosis de $0.02,0.05$ y $0.1 \mathrm{~g} / \mathrm{L}$. La reducción de la turbidez incluye también la remoción de microorganismos patógenos, donde el Azadirachta indica, logra disminuir el 99.4 y $99.2 \%$ de los coliformes totales y fecales, así como, el almidón de Zea mays, usado a concentración de $2.5 \mathrm{~g} / \mathrm{L}$ obtuvo una disminución del 94.4 y $83.6 \%$ respectivamente. Perú, país con especies multivariadas vegetales y animales, tiene un banco de compuestos orgánicos naturales, que pueden ser usados en el tratamiento de agua para consumo humano, sin embargo, no se han profundizado estudios con la vasta variedad de flora y fauna. La goma de tara es uno de los compuestos poco investigados en el tratamiento de agua para consumo humano y que por sus características fisicoquímicas puede ser un excelente ayudante de coagulación (De la Cruz, 2004).

La goma de tara es extraída de las semillas del árbol de tara (Caesalpinia spinosa), tiene alto peso molecular (aproximadamente 351400) (Siccha y Lock de Ugaz, citado por Goycochea, 2010), propiedades gelificantes y aglutinantes los cuales son importantes en polímeros utilizados como coagulantes o ayudantes de coagulación en el proceso de tratamiento de agua para consumo humano (Aguilar et al., 2014). Aguilar (2010) 
realizó una investigación usando goma de tara como ayudante de coagulación logrando reducir la turbidez del agua a valores de 1.7 y 1.5 UNT para muestras de 25 y 390 UNT respectivamente logrando reducir el uso de sulfato de aluminio tipo B (bauxita) hasta el 17\%. Con el propósito de profundizar el estudio de la goma de tara en tratamiento de agua para consumo humano, el objetivo de esta investigación fue determinar la influencia de la goma de tara como ayudante de coagulación en el proceso de coagulación-floculación para la remoción de turbidez de una suspensión artificial de bentonita.

\section{MATERIALES Y MÉTODOS}

Para las pruebas experimentales se ha utilizado goma de tara al $99 \%$ de pureza, proveniente de ANICOLSA del Perú S.A.C., y Sulfato de Aluminio tipo A de ARIS Industrial.

Suspensión de bentonita

Para la contaminación del agua fue utilizada la bentonita sódica, con características similares a la bentonita de Amotape (Piura). Vega et al., (1993) han determinado la composición química de la bentonita, utilizando el método de polvo cristalino de la muestra total, a través de un Difractómetro Philips PW 1710, con radiación Cu-Ka y operando a $40 \mathrm{KV}$ y $30 \mathrm{~mA}$. y que al diluir, por sus características químicas representan el sistema coloidal de las fuentes de aguas naturales (Tabla 1).

Tabla 1: Composición química de la bentonita de Amotape (Región Grau - Piura) Fuente: Vega et al., (1993)

\begin{tabular}{|l|l|}
\hline Elementos & \% en peso \\
\hline $\mathrm{SiO}_{2}$ & 66.0 \\
\hline $\mathrm{Al}_{2} \mathrm{O}_{3}$ & 12.51 \\
\hline $\mathrm{Fe}_{2} \mathrm{O}_{3}$ & 2.86 \\
\hline $\mathrm{Na} 2 \mathrm{O}$ & 0.43 \\
\hline $\mathrm{CaO}$ & 0.43 \\
\hline $\mathrm{MgO}$ & 1.93 \\
\hline $\mathrm{K} 2 \mathrm{O}$ & 0.37 \\
\hline $\mathrm{Ti}_{2} \mathrm{O}$ & 0.17 \\
\hline $\mathrm{P} 2 \mathrm{O} 5$ & 0.04 \\
\hline $\mathrm{LOI}$ & 14.02 \\
\hline
\end{tabular}

La suspensión de bentonita fue preparada en un recipiente, diluyendo arcilla en agua potable, se dejó reposar por 15 minutos, el sobrenadante (que contiene los coloides) fue retirado en otro recipiente, y se verificó la turbidez de las muestras, de 30 UNT y 400 UNT para cada caso, con un turbidímetro de mesa HANNA HI88703. Las muestras fueron almacenadas a temperatura ambiente por 24 horas para hidratación de la arcilla y el cloro residual no afecte al proceso. Asimismo, se midió el $\mathrm{pH}$ de cada muestra con ayuda de un $\mathrm{pH}$ metro portable HANNA HI-9025C

\section{Prueba de jarras}

Los ensayos de prueba de jarras fueron realizados con un equipo de seis reactores de la marca LOVIBOND ET750. Para la remoción de la turbidez se ha usado la metodología descrita por el Centro Panamericano de Ingeniería Sanitaria y Ciencias del Ambiente (De Vargas, 2004). Se preparó la muestra de agua contaminada (suspensión de bentonita) con valores de turbidez de 30 UNT y 400 UNT, que representaron aguas de baja y alta turbidez respectivamente. El equipo fue programado a 300 revoluciones por minuto para añadir el coagulante por 5 segundos aproximadamente, luego se disminuyó la velocidad de giro a 40 RPM por 20 minutos para favorecer la formación de flóculos. Finalmente, se detuvo el equipo, para el proceso de sedimentación por espacio de 10minutos.

Los sifones fueron construidos, según las recomendaciones del CEPIS (De Vargas, 2004). Se han extraído 6 muestras de 40 mililitros en vasos precipitados para medir la turbidez residual con el turbidímetro de mesa HANNA HI-88703. Para las pruebas con goma de tara, se añadieron las dosis de este ayudante de coagulación, 10 segundos antes que el sulfato de aluminio, siguiendo luego con el mismo procedimiento y los tiempos de acción. 


\section{Pruebas preliminares}

Los ensayos preliminares con sulfato de aluminio tipo A fueron realizados para obtener los valores de dosis y concentración óptimos, que sirven como base para los experimentos con goma de tara siguiendo la metodología usada por Aguilar (2010).

\section{Diseño experimental}

La distribución de los ensayos fue realizada de acuerdo al Diseño Central Compuesto con dos factores, la dosis de goma de tara al $0.1 \%$ y la dosis de sulfato de aluminio tipo A al $1 \%$. Los niveles codificados de cada factor se encuentran en la tabla 2. La variable respuesta fue la turbidez residual.

Se han realizado 13 ensayos (incluidos las tres repeticiones en el punto central). La tabla 3 muestra la distribución de los ensayos de laboratorio, en donde $\mathrm{X}_{1}$ y $\mathrm{X}_{2}$ representan las dosis de Goma de tara al $0.1 \%$ y sulfato de aluminio tipo $\mathrm{A}$ al $1 \%$ respectivamente. El análisis estadístico fue realizado con el software Statistica v.13.1

Tabla 2: Codificación de los factores de estudio con los niveles correspondientes

\begin{tabular}{|l|c|c|}
\hline \multirow{2}{*}{ Niveles } & \multicolumn{2}{|c|}{ Factores } \\
\cline { 2 - 3 } & $\mathrm{X}_{1}$ & $\mathrm{X}_{2}$ \\
& Dosis de goma de tara al $0.1 \%(\mathrm{mg} / \mathrm{L})$ & ${\text { Dosis } \mathrm{Al}_{3}\left(\mathrm{SO}_{4}\right)_{2} \text { al } 1 \%(\mathrm{mg} / \mathrm{L})}$ \\
\hline-1.42 & 0 & 15 \\
\hline-1 & 2.48 & 19.1 \\
\hline 0 & 8.48 & 29.1 \\
\hline 1 & 14.48 & 39.1 \\
\hline 1.42 & 16.96 & 43.25 \\
\hline
\end{tabular}

\section{RESULTADOS Y DISCUSIONES}

Las muestras de bentonita utilizadas para el estudio fueron de 30 UNT y 400 UNT, que representan aguas de turbidez baja y alta respectivamente. Similares muestras fueron realizadas por Aguilar, R. (2010) utilizando 25 UNT y 390 UNT en una suspensión de tierra común. Mientras que Zemmouri et al., (2011) trabajaron con soluciones de bentonita de 9 y 250 UNT. Las suspensiones de bentonita tienen un pH ligeramente alcalino de 7.81 a 8, encontrándose en un rango adecuado para el trabajo. Zemmouri et al., (2011) usando como contaminante a la bentonita determinó que los rangos próximos a pH neutros son óptimos en el tratamiento. Por tal motivo, no se modificó y se mantuvo el pH natural de cada muestra para todas las pruebas realizadas.

\section{Mecanismos de coagulación floculación inducidos por Sulfato de Aluminio tipo A}

El mecanismo de coagulación se desarrolla por la neutralización de la carga superficial del coloide (alta turbiedad) o por efecto barrido (baja turbiedad), que funciona por la formación de hidróxidos de aluminio que precipitan y arrastran los coloides formando flóculos al contacto entre estas partículas (Letterman et al., 1999). Estos mecanismos dependen de acuerdo a las concentraciones de coloides en el sistema. Mientras más concentración de contaminantes, el mecanismo que influye, en mayor parte, es por neutralización de cargas (Figura 1), mientras que para concentraciones bajas el mecanismo es la floculación por barrido (Figura 2).

La figura 3 muestra los resultados obtenidos de los ensayos de dosis óptima. La tendencia de la curva observada, para ambos casos, presenta el valor óptimo de dosis $(25 \mathrm{mg} / \mathrm{L})$ para lograr la turbidez residual más baja, se observa que, a menores y mayores dosis de $25 \mathrm{mg} / \mathrm{L}$, la remoción de turbidez no es tan eficiente. Esto se debe a que las partículas coloidales no han sido desestabilizadas por la falta de iones positivos de aluminio, por lo que aún quedan coloides estables. 


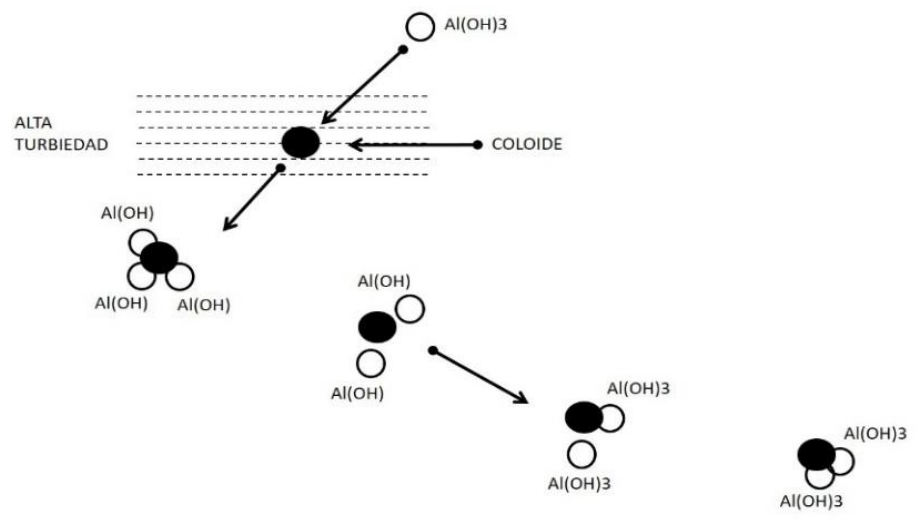

Fig. 1: Formación de flóculos por neutralización de carga, reacción rápida de $10^{-4}$ a 1 s (Andía, Y., 2010)

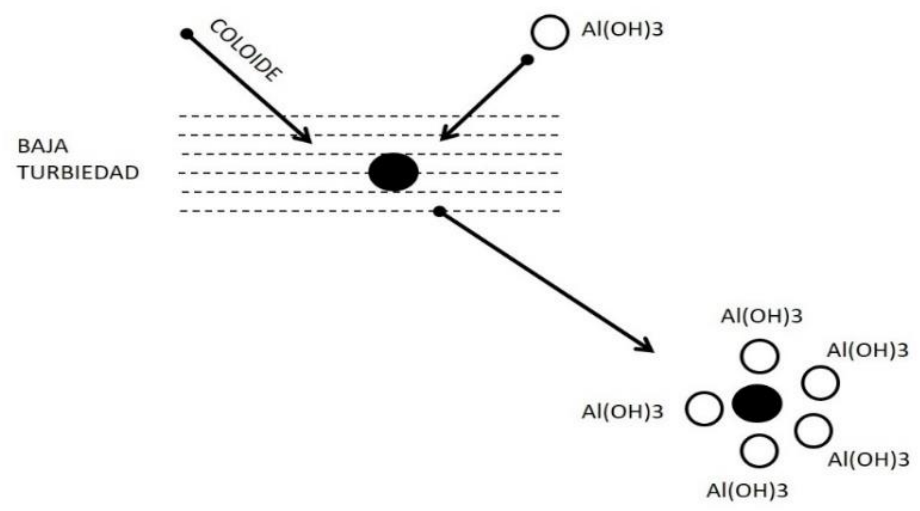

Fig. 2: Formación de flóculos por efecto barrido con dosis alta de sulfato de aluminio, reacción lenta 1 a 7 s (Andía, 2010)

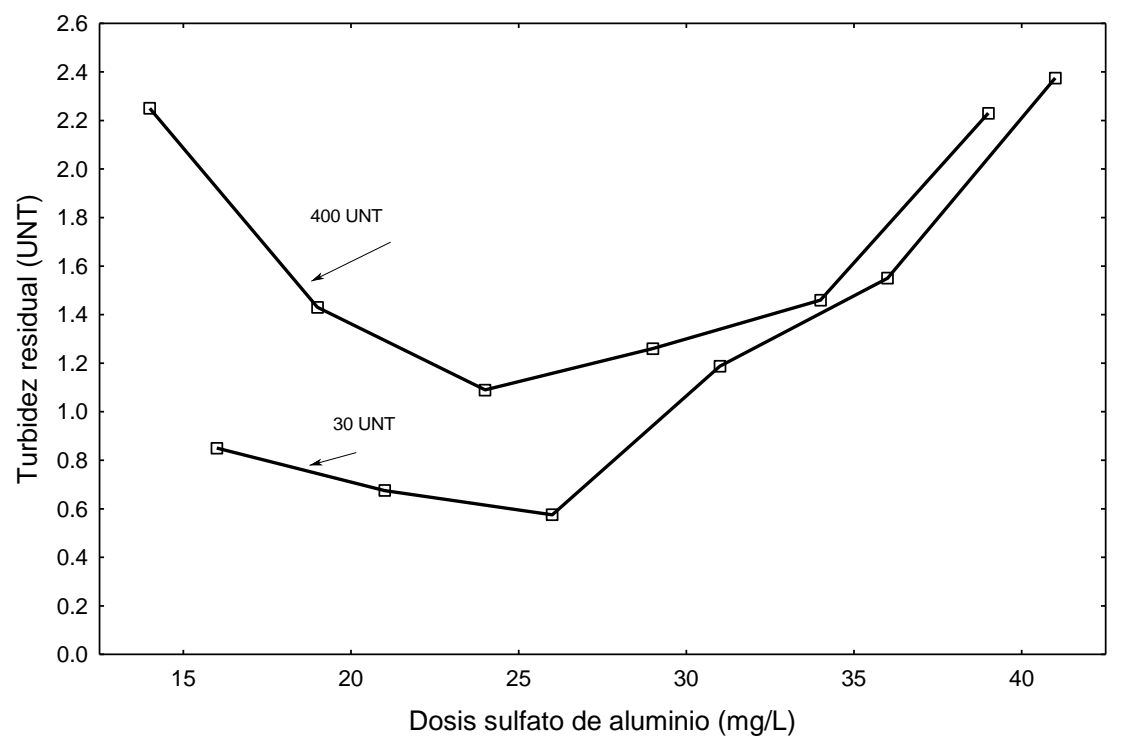

Fig. 3: Resultados de dosis óptima de sulfato de aluminio tipo $A$

El valor de turbidez residual se aproxima a 0.58 UNT para la muestra de 30 UNT a diferencia de 1.12 UNT para la muestra de alta turbidez. En el primer caso, el coagulante está funcionando mediante el mecanismo de coagulación por barrido, mediante la formación y posterior precipitación de hidróxidos de aluminio, mientras que en el segundo caso (muestra de 400 UNT) predomina el mecanismo de neutralización de cargas, provocando la desestabilización del coloide con carga negativa al contacto con los iones con carga positiva del aluminio y sus compuestos formados en el agua. El mecanismo de floculación por barrido, también puede arrastrar partículas como materia orgánica natural (Letterman et al., 1999). 
Luego de obtener la dosis óptima de sulfato de aluminio se procede a determinar la concentración óptima de este coagulante, mientras se mantiene constante la dosis de coagulante $(25 \mathrm{mg} / \mathrm{L})$ que se añade al sistema para ambos casos. La figura 4 muestra la concentración óptima para obtener la turbidez más baja (1\%) en ambos casos. Para la muestra con 400 UNT existe una tendencia bien marcada respecto a la concentración óptima, porque a menores y mayores valores de $1 \%$ no se logra la menor turbidez residual como sucede con el sulfato de aluminio al $1 \%$ con dosis de $25 \mathrm{mg} / \mathrm{L}$, como sucede con la muestra con 30 UNT a la concentración óptima de $1 \%$. De igual modo, se observa la disminución de turbidez residual a partir de valores de $5 \%$ hasta $10 \%$, llegando hasta una turbidez residual de 0.79 UNT. En términos de operación de plantas de tratamiento, la dilución de coagulantes a concentraciones altas puede favorecer a la formación de precipitados, que podrían obstruir los puntos de dosificación.

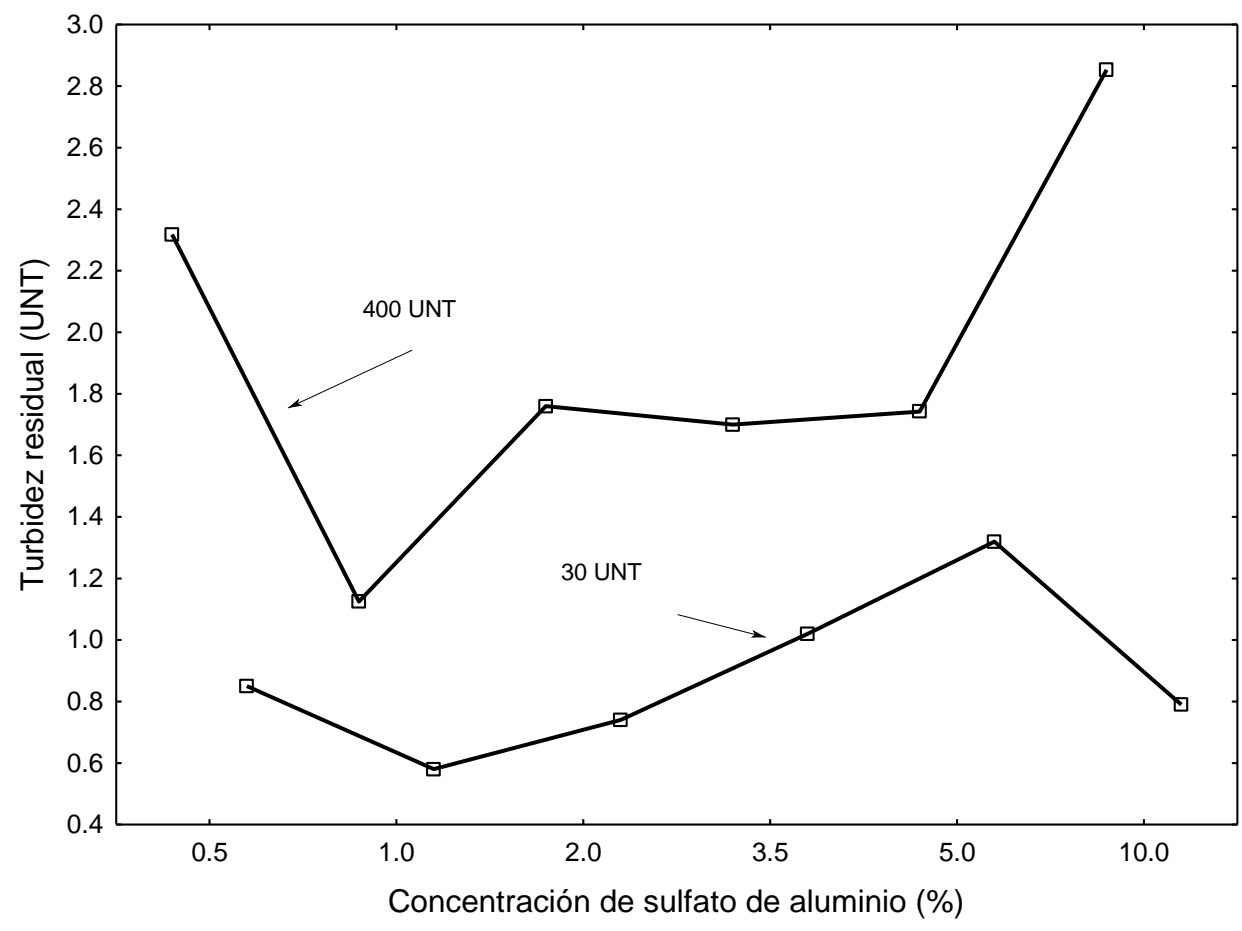

Fig. 4: Resultados de concentración óptima de sulfato de aluminio tipo $A$

Un estudio dirigido por Singley (1977) muestra que las mejores condiciones para la remoción de turbiedad se desarrollan cuando se utilizan soluciones de sulfato de aluminio bastante diluidas, sin embargo, con concentraciones inferiores a $0,1 \%$ la eficiencia en la remoción de turbiedad se reduce acentuadamente, debido a que el sulfato de aluminio puede hidrolizarse antes de su aplicación. Se sugieren valores del orden de 1 a $2 \%$ como ideales para adoptar en la operación de plantas tratamiento.

\section{Mecanismos de coagulación floculación inducidos por la goma de tara}

Con los resultados obtenidos del sulfato de aluminio, se determinó la dosificación de la goma de tara con los ensayos establecidos en la tabla 2. Aguilar, R. (2010), realizó ensayos de prueba de jarras utilizando dosis de goma de tara y reduciendo progresivamente las dosis de sulfato de aluminio tipo B. Las Figuras 5 y 6 muestran que el uso de la goma de tara como ayudante de coagulación ha logrado resultados de turbidez residual de 0.32 UNT para la muestra de 30 UNT y 0.40 UNT para la muestra de 400 UNT (Ver tabla 3).

La sustitución del sulfato de aluminio, en términos porcentuales representa un $40 \%$ para ensayos en la muestra de 30 UNT. Resultados similares fueron determinados por Hall (citado por Bolto, B. y Gregory, J., 2007), con el uso de polímeros naturales como ayudantes de coagulación ha reducido sustancialmente las dosis de sulfato de aluminio de 40 a $60 \%$, obteniendo iguales o mejores resultados en la remoción de la turbidez. Para el caso de la muestra de suspensión de bentonita con turbidez alta (400 UNT), la dosis de sulfato de aluminio usada no se ha reducido, manteniéndose en un valor aproximado a $25 \mathrm{mg} / \mathrm{L}$, sin embargo, al añadirle al sistema, la dosis de goma de tara al $0.1 \%$ (aproximadamente $12 \mathrm{mg} / \mathrm{L}$ ), la turbidez residual se ha reducido de 1.09 UNT (Valor obtenido en pruebas con sulfato de aluminio tipo A solamente) a 0.4 UNT, lo que representa una mejora del $63.3 \%$ en remoción de turbidez residual. 
Tabla 3: Resultados de turbidez residual (TR) expresados en Unidades nefelométricas de turbidez (UNT) de los ensayos de laboratorio usando goma de tara al $0.1 \%$ $\left(\mathrm{X}_{1}\right)$ y sulfato de aluminio al $1 \%\left(\mathrm{X}_{2}\right)$ tipo $A$ con repetición en el punto central $\left(^{*}\right)$

\begin{tabular}{|c|c|c|c|c|}
\hline Ensayo. & $\mathrm{X}_{1}$ & $\mathrm{X}_{2}$ & TR- 400UNT & TR- 30UNT \\
\hline 1 & -1 & -1 & 0.79 & 0.78 \\
\hline 2 & -1 & 1 & 1.09 & 1.20 \\
\hline 3 & 1 & -1 & 0.46 & 0.77 \\
\hline 4 & 1 & 1 & 0.77 & 1.44 \\
\hline 5 & -1.41 & 0 & 1.17 & 2.14 \\
\hline 6 & 1.41 & 0 & 0.58 & 1.27 \\
\hline 7 & 0 & -1.41 & 0.52 & 0.32 \\
\hline 8 & 0 & 1.41 & 2.14 & 1.86 \\
\hline 9 & 0 & 0 & 0.41 & 0.77 \\
\hline 10 & 0 & 0 & 0.47 & 0.68 \\
\hline 11 & $0^{*}$ & $0^{*}$ & 0.48 & 0.63 \\
\hline 12 & $0^{*}$ & $0^{*}$ & 0.40 & 0.68 \\
\hline 13 & $0^{*}$ & $0^{*}$ & 0.44 & 0.70 \\
\hline
\end{tabular}

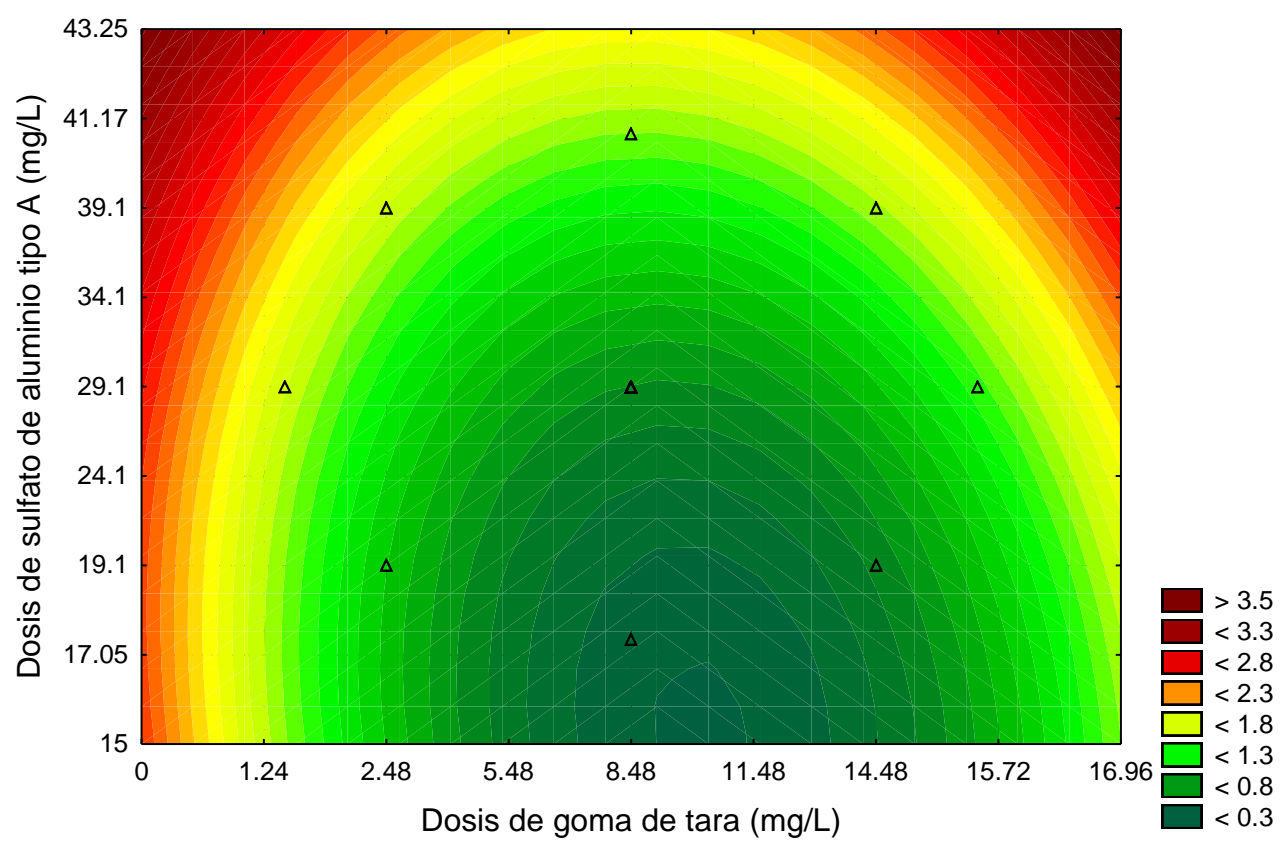

Fig. 5: Diagrama de contorno de turbidez residual para muestra a 30 UNT

La remoción de la turbidez con ayuda de la goma de tara corresponde a lo mencionado por Bolto et al., (2007), quienes sugieren que el mecanismo de floculación de partículas es por puentes poliméricos. Los polímeros por su larga cadena adhieren en su superficie los iones de aluminio y neutralizan la carga de los coloides. Este mecanismo hace que el tamaño de los flóculos sea más grande y sedimente con más facilidad. Shahriari, et al., (2011), usando mezclas de Plantago ovata y cloruro férrico, lograron una alta remoción de turbidez, cercano a 1 UNT en una escala de pH 8.04. De la misma forma, Laines et al., (2007) obtuvieron una eficiencia de remoción de turbidez con mezclas de almidón de plátano y sales de aluminio en un $98.6 \%$ para 126 UNT, a diferencia que el cuerpo de agua tratado fue lixiviado de relleno sanitario. Solís et al., (2012) trabajando con mezcla de almidón de yuca y sulfato de aluminio, logró porcentajes de remoción de turbidez de $97.9 \%$ (1.47UNT) para una muestra de 70 UNT.

Aguilar (2010) logró resultados de turbidez residual con el uso de la goma de tara como ayudante de coagulación valores de $1.5 \mathrm{UNT}$, con una reducción de la dosis de sulfato de aluminio tipo B de $5 \mathrm{mg} / \mathrm{L}$, que representa un ahorro de $16 \%$ de sulfato de aluminio, para una muestra de $390 \mathrm{UNT}$, mientras que, para una muestra de 25 UNT redujo en $2 \mathrm{mg} / \mathrm{L}$ la dosis de sulfato de aluminio (De $20 \mathrm{mg} / \mathrm{L}$ a $18 \mathrm{mg} / \mathrm{L}$ ) que representa la sustitución de un $10 \%$ del coagulante químico. 


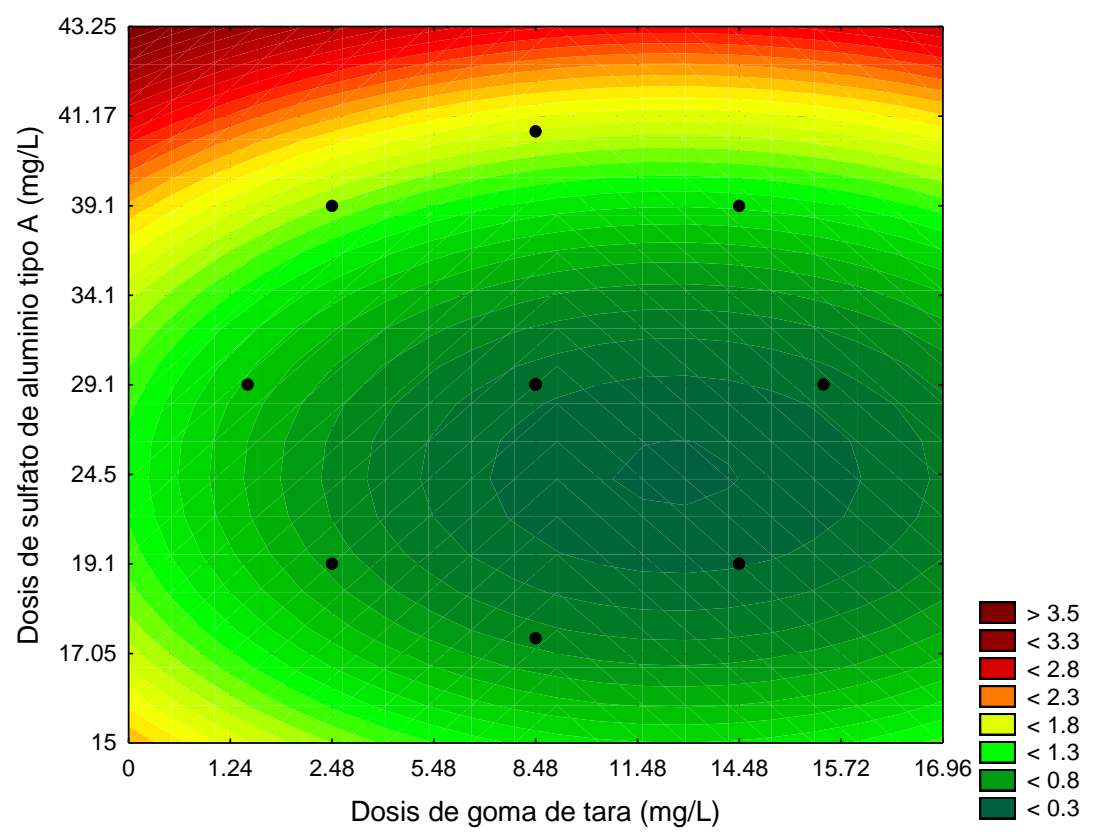

Fig. 6: Diagrama de contorno de turbidez residual para muestra de 400 UNT

\section{Análisis estadístico de ensayos con goma de tara y sulfato de aluminio}

La tabla 4 muestra el análisis de varianza para la remoción de turbidez residual de una suspensión de bentonita de 30 UNT y de 400UNT.

Tabla 4: Análisis de varianza de las variables independientes $\left(\mathrm{X}_{1}\right.$ dosis de goma de tara al $0.1 \% ; X_{2}$ dosis de sulfato de aluminio al $1 \%$ ) sobre la turbidez residual de agua sintética agua sintética a 30 UNT y 400 UNT

\begin{tabular}{|l|c|c|c|c|c|c|}
\hline \multicolumn{4}{|c|}{ Turbidez residual 30 UNT } & \multicolumn{3}{c|}{ Turbidez residual 400 UNT } \\
\hline & $\mathrm{SS}$ & $\mathrm{F}$ & $\mathrm{P}$ & $\mathrm{SS}$ & $\mathrm{F}$ & $\mathrm{P}$ \\
\hline $\mathrm{X}_{1}$ (lineal) & 0.125 & 1.307 & 0.290 & 0.275 & 3.360 & 0.109 \\
\hline $\mathrm{X}_{1}$ (cuadrático) & 1.221 & 12.763 & 0.009 & 0.129 & 1.575 & 0.249 \\
\hline $\mathrm{X}_{2}$ (lineal) & 1.334 & 13.950 & 0.007 & 1.051 & 12.835 & 0.008 \\
\hline $\mathrm{X}_{2}$ (cuadrático) & 0.086 & 0.903 & 0.373 & 0.920 & 11.230 & 0.012 \\
\hline $\mathrm{X}_{1}$ por $\mathrm{X}_{2}$ & 0.015 & 0.163 & 0.698 & 0.000025 & 0.00031 & 0.986 \\
\hline ERROR & 0.669 & & & 0.573 & & \\
\hline TOTAL SS & 3.389 & & & 2.877 & & \\
\hline
\end{tabular}

$$
\begin{aligned}
& T R_{30 U N T}=0.692-0.125 X_{1}+0.419 X_{1}^{2}+0.408 X_{2}+0.112 X_{2}^{2}+0.63 X_{1} X_{2} \\
& T R_{400 U N T}=0.44-0.186 X_{1}+0.136 X_{1}^{2}+0.363 X_{2}+0.364 X_{2}^{2}+0.0025 X_{1} X_{2}
\end{aligned}
$$

La ecuación 1 muestra el modelo de la turbidez residual (TR) para 30 UNT, con coeficiente de determinación de $\mathrm{R}^{2}=0.802$. Este modelo muestra que el componente cuadrático de la dosis de goma de tara al $0.1 \%\left(\mathrm{X}_{1}{ }^{2}\right)$ y el componente lineal de la dosis de sulfato de aluminio tipo $A$ en $1 \%\left(X_{2}\right)$ son significativos. Se observa que el componente lineal de la dosis de goma de tara no es significativo $\left(X_{1}\right)$, ni el componente cuadrático $\left(X_{2}{ }^{2}\right)$ de la dosis de sulfato de aluminio, así como las interacciones. Khanh, T. y L. Seok (2011) identificaron que la interacción entre la dosis de sal de aluminio y el pH, no es significativa para una muestra de agua de turbidez baja (7.5-8.5 UNT), sin embargo, la influencia del pH y de la dosis de la sal de aluminio, por separados, sí tuvieron efectos significativos.

La ecuación 2 muestra el modelo de la turbidez residual (TR) para 400 UNT, con coeficiente de determinación de $R^{2}=0.80$. Este modelo muestra que el componente lineal, cuadrático del modelo son significativos para el sulfato de aluminio tipo A. En estos ensayos, ni el componente lineal ni el cuadrático de la dosis de goma de tara influenciaron de manera significativa para la remoción de la turbidez. 


\section{CONCLUSIONES}

La utilización de goma de tara como ayudante de coagulación ayudó a reducir en $40 \%$ la dosis utilizada de sulfato de aluminio para la muestra de 30 UNT, también redujo la turbidez residual de 0.58 UNT a 0.32UNT.

La utilización de la goma de tara para muestra de 400 UNT no reduce la dosis de sulfato de aluminio, $\sin$ embargo, logró mejorar la remoción de la turbidez de 1.08 UNT a 0.4 UNT.

La goma de tara reduce su capacidad de coagulación cuando se incrementa la turbidez y es más efectiva en la reducción de uso de sulfato de aluminio cuando la turbidez del agua es menor.

\section{REFERENCIAS}

Abramovich, B., M. Lura y otros cuatro autores, Acción de Distintos Coagulantes para la Eliminación de Cryptosporidium spp. en el Proceso de Potabilización del Agua, Revista Argentina de Microbiología, ISSN: 0325-7541, 36, $92-96$ (2004)

Aguilar A., G. Noratto y otros tres autores, Potential of Tara (Caesalpinia spinosa) Gallotaninns and Hydrolyzates as Natural Antibacterial Compounds, doi: 10.1016/j.foodchem.2014.01.110, Food Chemistry, 156 (8), 301-305 (2014)

Aguilar, E., Utilización de las Semillas de Tara (Caesalpinia spinosa) como Ayudante de Coagulación en el Tratamiento de Aguas. Universidad Nacional de Ingeniería, Lima, Perú (2010)

Aguirre, S., N. Piraneque y R. Cruz, Sustancias Naturales: Alternativa para el Tratamiento de Agua del Río Magdalena en Palermo, Colombia, doi: 10.4067/S0718-07642018000300059, Inf. Tecnol., 29(3), 59-70 (2018)

Andía, Y., Tratamiento de Agua Coagulación y Floculación. Informe técnico Sedapal: Evaluación de plantas y desarrollo tecnológico, Lima, Perú (2000)

Almendárez, N., Comprobación de la Efectividad del Coagulante (Cochifloc) en Aguas del Lago de Managua "Piedras Azules", Revista Iberoamericana de Polímeros, ISSN-e: 0121-6651, 5(1), 46-54 (2004)

Barrenechea, A., Tratamiento de Agua para Consumo Humano. Plantas de Filtración Rápida. Manual I. Coagulación. Capítulo 4, Centro Panamericano de Ingeniería Sanitaria y Ambiental. Organización Panamericana de la Salud (2004)

Becaria, A., D. Lahiri y otros cinco autores, Aluminum and Copper in Drinking Water Enhance Inflammatory or Oxidative Events Specifically in the Brain, doi:10.1016/j.jneuroim.2006.03.025. Journal of Neuroimmunology, 176, 16-23 (2006)

Bolto, B. y J. Gregory, Organic Polyelectrolytes in Water Treatment, doi:10.1016/j.watres.2007.03.012, Water Research, 41, 2301-2324 (2007)

De La Cruz, P. Aprovechamiento Integral y Racional de la Tara (Caesalpinia spinosa-Caesalpinia tinctoria). Revista del Instituto de Investigación FIGMMG, UNMSM, ISSN-e:1561-0888, 7(14), 64-73 (2004)

De Vargas, L., Criterios para la Selección de los Procesos y de los Parámetros Óptimos de las Unidades. Tratamiento de Agua para Consumo Humano. Plantas de Filtración Rápida, Manual I, Capítulo 11, Centro Panamericano de Ingeniería Sanitaria y Ambiental, Organización Panamericana de la Salud (2004)

Di Bernardo, A. y L. Di Bernardo, Uso de Amido de Mandioca Catiônico como Auxiliar de Floculação, Escola de Engenharia de São Carlos EESC/USP (2002)

Díaz, C., C. Fall y otros seis autores, Agua Potable para Comunidades Rurales, Reúso y Tratamientos Avanzados de Aguas Residuales Domésticas, Red lberoamericana de Potabilización y Depuración del Agua RIPDA, Centro Interamericano de Recursos del Agua CIRA de la Facultad de Ingeniería de la Universidad Autónoma del Estado de México UAEM (2007)

Gauthier, E., I. Fortier y otros cuatro autores, Aluminum Forms in Drinking Water and Risk of Alzheimer's Disease, doi:10.1006/enrs.2000.4101, Environmental Research Section A, 84, 234-246 (2000)

Goycochea, R., Evaluación de Taninos y Goma del Fruto de la Tara Caesalpinia spinosa (Molina) Kuntze Provenientes de las Lomas de Atiquipa, Arequipa - Perú, Facultad de Ciencias Forestales, Universidad Nacional Agraria La Molina. Lima, Perú (2010)

Jia, J., The Role of Coagulation in Water Treatment, doi: 10.1016/j.coche.2015.01.008, Chemical Engineering, 8 , 36-44 (2015)

Joshi, J. y O. Sahju, Azadirachta indica Leaves as Antibacterial Treatment on Drinking Water, doi:10.12691/ijcn-2-2-3, International Journal of Clinical Nutrition, 2(2), 36-40 (2014)

Katrivesis F.K., A.D. Karela, V.G. Papadakis y C.A. Paraskeva, Revisiting of Coagulation-Flocculation Processes in the Production of Potable Water, doi: 10.1016/j.jwpe.2018.12.007, Journal of Water Process Engineering, 27, 193-204 (2019)

Khanh, T. y L. Seok, Response Surface Methodological Approach to Optimize the Coagulation - Flocculation Process in Drinking Water Treatment, doi:10.1016/j.cherd.2010.12.004, Chemical Engineering Research and Design, 89, $1126-1135(2011)$ 
Kirchmer, C., J. Arboleda y M. Castro, Polímeros Naturales y su Aplicación como Ayudantes de Floculación, Centro Panamericano de Ingeniería Sanitaria Ciencias del Ambiente (CEPIS), Lima, Perú (1975)

Laines, J., J. Goñi, R. Adams y W. Camacho, Mezclas con Potencial Coagulante para Tratamiento de Lixiviados de un Relleno Sanitario, Revista Interciencia, ISSN-e: 2244-7776, 33(1), 22-28 (2007)

Letterman, R., A. Amirtharajah y C. O'melia, Coagulation and Flocculation. Water Quality and Treatment, $5^{\text {th }}$ Ed. McGrawHill Inc., New York, Department of Civil and Environmental Engineering, Syracuse University, Syracuse, New York, School of Civil and Environmental Engineering, Georgia Institute of Technology. Department of Geography and Environmental Engineering, the Johns Hopkins University, Baltimore, Maryland, Atlanta, Georgia (1999)

Matilainen, A., M. Vepsäläinen y M. Sillanpää, Natural Organic Matter Removal by Coagulation During Drinking Water Treatment: A review, doi: 10.1016/j.cis.2010.06.007, Advances in Colloid and Interface Science, 159, 189-197 (2010)

Mendoza, I., L. Fuentes y otros cinco autores, Eficiencia de Hylocereus lemairei como Coagulante-Floculante en Aguas para Consumo Humano, Revista arbitrada venezolana del Núcleo LUZ-Costa Oriental del Lago, ISSN: 1836-5042, (1), 5369 (2007)

Organización Mundial de la Salud (OMS), Drinking-Water Quality and Preventing Water-Borne Infectious Disease, Water Sanitation and Health (WSH), WHO (2012)

Preston, K., D. Lantagne, N. Klotarz y K. Jellison, Turbidity and Chlorine Demand Reduction Using Alum and Moringa Flocculation Before Household Chlorination in Developing Countries, doi:10.2166/wh.2009.210, Journal of Water and Health, 8(1), 60-70 (2010)

Restrepo, O., Evaluación Del Proceso de Coagulación-Floculación de una Planta de Tratamiento de Agua Potable, Tesis de grado, Facultad de Minas, Universidad Nacional de Colombia, sede Medellín, Colombia (2009)

Shahriari, T., G. Nabi y Sh. Shahriari, Evaluating the Efficiency of Plantago Ovata and Starch in Water Turbidity Removal, Doi:10.22059/IJER.2011.491, International Journal of Enviroment Research, 6(1), 259-264 (2011)

Singley, J., Revisión de la Teoría de la Coagulación del Agua, Simposio sobre Nuevos Métodos de Tratamiento de Agua, Departamento de Ingeniería Ambiental, Universidad de Florida, Gainesville Florida, EE.UU. (1977)

Solís, R., J. Laines y J. Hernández, Mezclas con Potencial Coagulante para Clarificar Aguas Superficiales, Rev. Int. Contam. Ambie., ISSN: 0188-4999, 28(3), 229-236 (2012)

Trujillo, D., L. Duque y otros cuatro autores, Remoción de Turbiedad en Agua de una Fuente Natural Mediante Coagulación/Floculación Usando Almidón de Plátano, Rev. ion, ISSN 0120-100X, 27(1), 17-34 (2014)

Vega, J., L. Verdeja, M. Sancho y J. Garcia, Caracterización y Propiedades de las Bentonitas de Amotape (Región GrauPerú), Bol. Soc. Esp. Ceram. Vidr., 32 (6), 377-383 (1993)

Wicklund, A., A. Sparen y otros tres autores, Bioavailability of Labile Aluminium in Acidic Drinking Water: A Study in the Rat, doi: 10.1016/0278-6915(95)00002, J. Food and Chemical Toxicology, 33(5), 403-408 (1994)

Yongabi, K.A., Biocoagulants for Water and Waste Water Purification: a Review, International Review of chemical engineering, ISSN-e: 2533-1760, 2(3), 444-458 (2010)

Yu, Y., Y. Hun y otros tres autores, Multi-Barrier Approach for Removing Organic Micropollutants Using Mobile Water Treatment Systems, doi: 10.1016/j.scitotenv.2018.05.079, Science of the Total Environment 639, 331-338 (2018)

Zemmouri, H., S. Kadouche y otros tres autores, Use of Chitosan in Coagulation Flocculation of Raw Water of Keddara and Beni Amrane dams, doi:10.2166/ws.2011.038, Water Science y Technology: Water Supply, 11(2), 202-210 (2011) 